\title{
Tinnitus, Hyperacusis, and Misophonia Toolbox
}

\author{
Jill B. Meltzer, Au.D. ${ }^{1}$ and Melanie Herzfeld, Au.D. ${ }^{2}$
}

ABSTRACT

The management of tinnitus, hyperacusis, and misophonia can be a challenge to the audiologist, physician, and psychologist, as well as the patient and their family. Assisting the patient in identifying cognitive distortions or misperceptions can aid in the transition from one who suffers from these conditions to one who is able to manage them. Although there is no cure, it is inappropriate to tell patients that nothing can be done. The audiologist can help the patient learn techniques to lower the emotional impact of tinnitus. For those with an interest in tinnitus and sound sensitivity management, many tools are available. Most importantly, knowing when to refer is paramount. Compiling a referral list of medical and allied health providers, as well as audiologists who specialize in tinnitus, hyperacusis, and misophonia management, is in the best interest of the patient. Being familiar with the ever increasing list of available products and devices is necessary to build the tinnitus toolbox. This article will review current treatments, trends, and tools that may assist in patient education and tinnitus habituation. Some attention also will be paid to other sound sensitivities such as hyperacusis and misophonia.

KEYWORDS: Tinnitus, hyperacusis, misophonia

Learning Outcomes: As a result of this activity, the participant will be able to (1) compare multiple tinnitus treatments that are currently available and (2) list several devices available for tinnitus management.

A germinal article by Heller and Bergman investigated the presence of tinnitus in normalhearing subjects. ${ }^{1}$ Although $\sim 73 \%$ of their subjects with hearing loss reported some tinni- tus, $94 \%$ of the normal-hearing subjects reported tinnitus when the environment was quiet enough. The consensus of these authors was that tinnitus is likely to be subaudible but
${ }^{1}$ North Shore Audio-Vestibular Lab, Highland Park, Illinois; ${ }^{2}$ Hearing and Tinnitus Center, Woodbury, New York.

Address for correspondence: Melanie Herzfeld, Au.D., Hearing and Tinnitus Center, 113 Crossways Park Dr. \#101, Woodbury, NY 11797

(e-mail: mherzfeld@earminder.com).
Tinnitus, Hyperacusis, and Misophonia; Guest Editors, Diane F. Duddy, Au.D. and Laura A. Flowers, Au.D.

Semin Hear 2014;35:121-130. Copyright (C) 2014 by Thieme Medical Publishers, Inc., 333 Seventh Avenue, New York, NY 10001, USA. Tel: +1(212) 584-4662. DOI: http://dx.doi.org/10.1055/s-0034-1372528. ISSN 0734-0451. 
present in almost everyone and therefore could not be eliminated. Making tinnitus subaudible would therefore be the goal of tinnitus management. ${ }^{1}$ From this perspective, audiologists use the management techniques discussed in this article to aim for the subaudible status Heller and Bergman referenced.

"A strong case can be made that audiologists are in the best position of all healthcare professionals to serve as the primary treatment provider for patients with tinnitus." ${ }^{2}$ First, audiologists fit hearing aids that are often an effective first step in tinnitus management. When hearing loss is present and tinnitus is reported, hearing aids may serve a double benefit of improved communication and tinnitus reduction. ${ }^{3}$ Second, audiologists routinely counsel patients about hearing loss and listening strategies to improve communication skills. Counseling plays a significant role in most successful tinnitus management programs. ${ }^{4,5}$ Directive counseling is a key aspect of Tinnitus Retraining Therapy (TRT); other tinnitus management strategies advocate collaborative or patient-centered counseling. ${ }^{6}$

How successful can audiological tinnitus management be? In a study by Herzfeld and $\mathrm{Kuk},{ }^{7}$ as many as $90 \%$ of patients who had hearing loss were able to significantly reduce their tinnitus reaction by using combination amplification and tinnitus devices. Other studies have shown significant reductions in tinnitus handicap or tinnitus reaction ranging from 60 to $85 \%$ with the use of counseling, TRT, Neuromonics Tinnitus Treatment (Neuromonics, Inc., Westminster, $\mathrm{CO}$ ), sound generators, and even hearing aids. ${ }^{8}$ The definition of success in most studies is a 20 -point difference or $\sim 40 \%$ reduction on a tinnitus inventory comparing pretreatment to posttreatment scores. ${ }^{8}$

Sound generators or maskers are not new. As Hippocrates noted in $400 \mathrm{BC}$, the "greater sound drives out the less" in discussing why buzzing seemed to be lessened in the presence of external sounds. In 1976, Jack Vernon may have been one of the first to advocate wearable masking devices to aid in tinnitus management. ${ }^{9}$ Although allowing the noxious sound of tinnitus to be masked using broadband white noise, questions arose regarding whether habituation, the ultimate goal, was possible. Habit- uation is best defined as the lack of a response to a stimulation; to be unaware of tinnitus unless one decides to look for it. For some patients, the loudness of the masker became a secondary disturbance and thus maskers fell out of favor for some years. Recent masking paradigms have trended more toward partial masking. In TRT, for example, Jastreboff and Hazell recommend using the mixing point, or the point where one hears the tinnitus change because of the presence of a mingling sound. ${ }^{10}$ Tyler et al suggest that the use of maskers set to the mixing point may not be the only method to achieve habituation. ${ }^{11}$ Their study showed no significant difference between three groups-one group receiving counseling and utilizing maskers set to the mixing point, one group using full masking along with counseling, and one group receiving counseling alone. Although the number of subjects in this study was small, it is noteworthy that the groups with the greatest degree of positive change on inventories were the groups who had counseling and masking set to the mixing point used in tandem, even if the numbers were not reaching significance. ${ }^{11}$ Time will tell if full masking regains the confidence and recommendations of audiologists and tinnitus patients.

Goodey states that we do not really manage tinnitus, rather we manage the patient who has tinnitus. ${ }^{12}$ He has suggested that there are three categories of tinnitus treatment. The first category is aiding the input, or auditory side, to normalize sensory inputs for the patient. This includes correcting any auditory loss and again points to the use of amplification to offer the patient an enriched auditory environment. Think back to the original study of Heller and Bergman ${ }^{1}$; in a sound-treated environment where the noise floor was so low, the normalhearing subjects heard their tinnitus. Once out of that special environment, they did not. Similarly, if we surround the area of tinnitus with sound via amplification, a large number of tinnitus patients will immediately hear less tinnitus. This is not aiming toward habituation, only reducing the time tinnitus is heard.

The second category Goodey discusses is controlling the emotional factors. ${ }^{12}$ It cannot be stressed enough that emotional factors are cited in virtually every tinnitus management 
program, as it is the emotional response that drives the tinnitus into the disturbance area. Consider the auditory mechanism as a go/no go type of system. In most cases of tinnitus, there is an excess of energy transmitted. The subcortex or cortex then has a choice to acknowledge the tinnitus or ignore it. Once it is acknowledged, the next level of go/no go is whether or not the tinnitus is bothersome or just present. If bothersome, the limbic system and autonomic nervous system become involved. Reduction of the emotional impact of tinnitus and ultimately of the tinnitus perception is the basis of most tinnitus management systems, ${ }^{13}$ whether one considers directive counseling via TRT, in which one learns information about tinnitus and why one's emotional response drives the tinnitus to the forefront, ${ }^{14}$ or traditional cognitive behavioral therapy (CBT), ${ }^{15}$ or even cognitive behavioral integration $(\mathrm{CBI})$ as promulgated by Sweetow. ${ }^{16}$ In all cases, the goal is behavior management and change. Think of tinnitus and the brain as a Pavlovian model. If the brain looks at tinnitus as a negative event, then it will cause a reaction each time it is acknowledged. It is difficult to extinguish this conditioned response if it has a negative connotation. Thus, the goal of the counseling portion of tinnitus management will revolve around changing behaviors and attitudes. ${ }^{15}$

The third category Goodey references is the use of what he terms "direct approaches to the central nervous system." 12 Neuroplastic changes can be influenced by the use of medications, dietary modifications, electrical stimulation, magnetic stimulation, and even surgical considerations. He suggests that even if these things can foster neuroplastic changes, they require influences of psychological and sensory inputs. $^{12}$

\section{TINNITUS AND SOUND SENSITIVITY MANAGEMENT METHODS}

The main goal of tinnitus management using sound therapy is to lower the strength of the tinnitus signal. If we increase the level of surrounding sound, we frequently make the tinnitus appear lower. ${ }^{10}$ Picture a candle in a dark room versus a candle in a lit room. Although the candle is equally bright in both scenarios, we typically judge its impact as less in a lit room. Thus, by surrounding the tinnitus sound with other sounds, it may be described as less loud or noticeable as in a quiet room.

As mentioned previously, audiological management of tinnitus should always include some aspect of counseling. The counseling commences as soon as the appointment begins. At its core, counseling can be thought of as education. Being an informed, but empathetic listener and reassuring the patient that others have experienced disturbing tinnitus and have found relief provides hope. Counseling may help the patient understand their tinnitus, enabling a reduction of fear and distress. ${ }^{17}$ Negative counseling can have disastrous effects on the person suffering from tinnitus. Numerous times patients have gone to their physician and/or audiologist with a primary complaint of tinnitus only to be told, "There's nothing you can do about it, just live with it, it's incurable," and they leave more distressed. Stephen $\mathrm{Na}$ gler, ${ }^{18}$ Medical Director of General Hearing Instruments, says it well in his "Patient Perspective." In this document, he states that a physician can take 5 minutes and either tell the patient there is no cure or instead take the time to say that although there is no cure, there are professionals with an interest in tinnitus who may be able to help them. Offering hope, reassurance, and empowerment to the patient is a significant first step, fostering confidence in becoming the manager of the tinnitus and not the victim of it.

\section{Cognitive Behavioral Therapy}

CBT addresses some of the automatic negative thoughts associated with tinnitus perception. Automatic thoughts can be neutral, negative, or positive. In CBT, the goal is not to change the actual perception of the tinnitus but instead to acknowledge and alter the maladaptive thoughts and reactions to the tinnitus. ${ }^{15}$ In his "Letter to a Tinnitus Sufferer," Nagler referred to these as cognitive distortions and counseled the tinnitus sufferer to practice and pay attention to these distortions, as they are the basis of finding relief. ${ }^{19} \mathrm{CBT}$ often uses an 
"A-B-C" model: $A$ is the situation or event that leads to the thoughts and beliefs about the event, or $B$. The thoughts lead to $C$, the emotional consequences. Henry and Wilson devote an entire chapter in their book to the connection between thoughts and emotions. ${ }^{20}$ CBT teaches the patient to identify, evaluate, and respond to their dysfunctional thoughts and beliefs, thereby educating in a collaborative way, to change thinking, mood, and behavior. ${ }^{5}$ The actual perception of the tinnitus is secondary to the severe negative reaction to the tinnitus.

Additionally, the patient who is constantly monitoring their tinnitus will never find relief. For some, the continuing questioning of "How long until I habituate" or "Must I still wear my devices?" indicates that the patient is still actively monitoring and tinnitus will continue to be a problem. ${ }^{21}$ The more a person "visits" or "listens in" for their tinnitus, the more likely they will find it. Equally counterproductive are the patients who spend every waking moment scouring the Internet searching for any and all sources related to tinnitus. It is important to tell these patients that too much time spent investigating tinnitus is not helpful in tinnitus management. To address excessive patient monitoring and investigation of tinnitus, distraction techniques are another essential part of CBT. A person can be very aware of any number of sensations if they turn their attention to it. Feeling my feet in my shoes, my ring on my finger, my watch on my arm are sensations available to me, but these are not sensations that demand attention. Pointing these out to the patient can be helpful exercises in redirecting attention away from the tinnitus. The importance of being able to refocus, reimage, and reimagine is vital. Finally, learning the guidelines of relaxation, deep breathing, and guided imagery is a necessary component of this therapy.

\section{Mindfulness-Based Stress Reduction}

Mindfulness therapy is another counselingbased therapy that may be helpful for tinnitus patients. With mindfulness-based stressed reduction, one pays attention to thoughts and sensations without judgment of those thoughts and sensations. In Western culture, Jon KabatZinn is credited with bringing mindfulness to today's modern medicine. He founded the mindfulness-based stressed reduction program at the University of Massachusetts Medical Center. Mindfulness has found its way into tinnitus treatment. Psychologist Jennifer Gans has used it in a pilot program entitled "Mindfulness Based Tinnitus Stress Reduction." ${ }^{22}$

\section{Tinnitus Retraining Therapy}

TRT, as described and implemented by Jastreboff and Hazell, ${ }^{10}$ is based on a neurophysiological model of tinnitus and has applications in tinnitus, hyperacusis, and misophonia treatment. The therapy depends on brain neuroplasticity and the ability of the brain to habituate to unimportant signals. TRT assumes that tinnitus is a phantom perception and the distress from tinnitus includes nonauditory brain networks such as the limbic and autonomic nervous systems. Therefore, the auditory system is secondary for clinically relevant tinnitus. In TRT, the counseling aspect attempts to reclassify the tinnitus as a neutral stimuli instead of a negative one. If the tinnitus is judged to be neutral, the degree of tinnitus reaction is lessened and habituation is more likely. ${ }^{10}$

According to Jastreboff and Hazell, ${ }^{10}$ hyperacusis results from increased gain in the auditory pathways and is determined by the sound characteristics; the character or meaning of the sound is irrelevant. It is simply that the loudness of the sound is uncomfortably loud (and therefore troublesome), as determined by loudness discomfort levels. The limbic system is not necessarily involved. Hyperacusis should in actuality be considered before tinnitus management. It is impossible to effectively manage tinnitus if the sound level of the masker or sound generator is too loud for the patient to tolerate. ${ }^{10}$

TRT addresses another sound sensitivity condition termed misophonia. Misophonia differs from true hyperacusis in that the limbic and autonomic nervous systems are integral components of the negative feedback loop. Its definition was first promulgated by Jastreboff and Hazell just over a decade ago. ${ }^{10}$ Another term frequently associated with misophonia is 
selective sound sensitivity syndrome, introduced by Portland audiologist, Marsha Johnson. Misophonia is a reaction to sounds, not relative to loudness, but instead to the emotional impact of specific sounds. In misophonia, specific sounds, such as chewing, eating and mouth sounds, breathing, tapping or clicking, or even repetitive visual triggers, elicit a severe negative emotional and physical reaction, which is commonly anger and disgust. We often refer to these debilitating sounds as trigger sounds. Misophonia engages the limbic system in a powerful, negative way and a conditioned reflex is established. Loudness discomfort levels are often normal in the misophonic patient. Associating the disliked sound with something positive is the fastest way to reverse the conditioned response. ${ }^{4}$ By giving the patient a chance to identify the disturbing sound as a pleasant one, we can help the misophonic patient learn to reverse the reflex. For example, if the sound of a crunching potato chip (trigger sound) is mixed with the sound of a campfire on a beach (pleasant association), or if the sound of clipping nails (trigger sound) is thought instead as the sound of horses clopping through the park or the sound of roses being clipped (pleasant association), then the association can be made less unpleasant. Think of it as a pain-management technique. One does not need to experience needles to learn how to cope with injections, but instead would benefit from learning how to focus away from the pain. Those of us who have worked with misophonic patients have found this to be true.

According to the TRT model, treatment for hyperacusis and misophonia must be different as the role of the limbic system and autonomic nervous system are not the same.

\section{Progressive Tinnitus Management}

The Veterans Administration reports a large number of veterans with service-connected tinnitus. As a result, Henry et al produced and published the Progressive Tinnitus Management (PTM) workbook with accompanying videos. ${ }^{6} \mathrm{PTM}$ is available as a member benefit of the American Tinnitus Association (ATA) or through online booksellers. As part of the PTM approach, the patient with tinnitus is shown deep breathing exercises, progressive relaxation, and guided imagery. The patient is instructed to find environmental sounds, such as music or speech, that fall into the categories of soothing, background, and interesting. By rating these sounds on a success scale, the patient can find sounds that help soothe, distract, engage, or work as a background sound. This Veterans Administration step-management approach starts with a group encounter to help the veteran learn how to fill out the sound-rating sheets to find sounds that are beneficial. The veteran returns a few weeks later for a discussion of how beneficial the sound files were for their tinnitus. For many, these group sessions provide sufficient help in tinnitus management. Further help is given for those who need amplification, have additional counseling needs, and require individual sessions. ${ }^{6}$ The ATA supports local group meetings that allow tinnitus patients to meet one another, to share stories, to share successes and failures, and to learn about tinnitus management options and research.

\section{Tinnitus Activities Treatment}

Tyler and colleagues at the University of Iowa provide online tools as part of the Tinnitus Activities Treatment program to assist the audiologist in tinnitus management. ${ }^{23}$ Through the university's Web site (see Appendix A), the tinnitus patient is offered downloadable questionnaires to identify specific areas of tinnitus impact, surveys to describe their tinnitus experience, and directions to participate in tinnitus and hyperacusis trials. The Web site also offers counseling presentations covering thoughts and emotions, hearing and communication, sleep, concentration, and different sound therapies available for tinnitus management. These presentations are both helpful for the tinnitus patient as well as the audiologist working with the tinnitus patient. ${ }^{23}$

\section{ASSESSMENT AND MANAGEMENT}

\section{Building the Tinnitus Toolbox}

When evaluating the tinnitus population, the importance of assessing the impact of tinnitus 
cannot be overstated. There is no direct correlation between the psychoacoustic characteristics of tinnitus (pitch, loudness, sound quality, etc.) and the degree of reaction. ${ }^{10}$ Therefore, it is very important to consider the areas of greatest impact; including when and how tinnitus interferes. There are inventories/questionnaires that can assist the audiologist in first assessing the level of tinnitus disturbance or handicap and subsequently monitor treatment progress. The Tinnitus Handicap Inventory is a 25 -item questionnaire, whereby the patient answers yes, no, or sometimes to each item. The Tinnitus Handicap Inventory is easily scored and can give a quick assessment of how the tinnitus impacts daily activities. ${ }^{24}$ The Tinnitus Reaction Questionnaire is a 26-item inventory to assess tinnitus distress levels, asking the patient to rate each item from 0 (not at all) to 4 (all the time). ${ }^{25}$ The Iowa Tinnitus Handicap Questionnaire was developed in 1990 by Kuk et al and has 27 questions. ${ }^{26}$ Answers to the items range from 0 (strongly disagree), to 100 (strongly agree). The Tinnitus Functional Index assesses eight aspects of tinnitus: intrusiveness, concentration, sleep, sense of control, cognitive, auditory, relaxation, and quality of life. ${ }^{27}$ These are but a few of the currently validated tools that may be used to measure and monitor tinnitus outcomes.

What kind of management strategies can be implemented for those with tinnitus and other sound sensitivity disorders? The distress level (as indicated by the inventory and/or tinnitus history) will dictate the aggressiveness of the treatment. Many people experience tinnitus in a form they would consider acceptable, whereas others consider their tinnitus to be completely unacceptable and are severely debilitated. Some find the level of tinnitus disturbance to be minimal. For them, reassurance and some minor sound therapy is often enough. One can use ear-level devices or environmental sounds to help reduce tinnitus awareness. For others, the level of disturbance is higher and these patients may need more counseling or a referral to a psychologist, social worker, or psychiatrist. The value of referral lists cannot be overstated. Although we may be trained in counseling, we also need to know when the needs of the patient exceed our scope of practice.

Tinnitus often disrupts concentration and sleep. Sleep disturbances are often cited as one of the comorbidities of tinnitus. According to Cronlein et $\mathrm{al}^{28} 45 \%$ of patients with new tinnitus report sleep disturbance. Some mourn the loss of a quiet night of sleep, others lament the inability to sleep without awakening in the middle of the night. For many patients, addressing the sleep-deprivation issue can be a good start to managing tinnitus. There are products specifically designed for sleep management, such as sound pillows, pillow speakers, Bedphones, and SleepPhones, which deliver sound at ear level throughout the night. In addition, the sound source may be emitted from a cell phone through a noise app, tablet, or from a tabletop sound machine. Certain medications, such as benzodiazepines or other sleep-inducing products, may be prescribed for sleep management. However, the sooner sleep problems are addressed and tinnitus management implemented, the less likely the patient is to seek drug therapy. TRT suggests avoidance of benzodiazepines, because they slow the progress toward habituation and alter perception. ${ }^{10}$ Ultimately, it is recommended that patients seek physician advice regarding appropriate medications for sleep.

Somatosensory inputs can alter the level of tinnitus, whether from temporomandibular joint disorders, neck involvement, or myofascial triggers. Thus, patients may complain of neck and/or back issues or jaw clenching and teeth grinding. Surprisingly, just knowing that this may be the source of the problem relieves some of the patient's anxiety. It is beneficial to build a referral list of physicians and dentists who specialize in these issues, as treatments for neck, back, or temporomandibular joint disorders may help alleviate tinnitus. For example, patients may be told to change their pillow array, or perhaps to sleep with a wedge to change the body alignment at night. Some patients benefit from physical therapy or have received injections in specific sites as a result of these referrals. Understanding the need for and providing referrals for these specific issues may improve the success of the tinnitus-management program. 


\section{Tinnitus Devices}

There are specific treatments and devices that have been designed, marketed, and approved for tinnitus management that will be discussed, such as Neuromonics Tinnitus Treatment, the SoundCure Serenade device, and the PAXX tinnitus device. This is not an endorsement of a particular management option; some may be more beneficial than others. Each option is a tool developed with the goal of tinnitus habituation, relief, and management. As mentioned previously, hearing aids, sound generators, or combination devices also may be very effective options. Currently, many of the hearing aid manufacturers have combination amplification and tinnitus devices, and the options are rapidly increasing. One can now choose from fractal music and relaxing tones, white noise, pink noise, frequency-shaped noise, narrowband noise, as well as streaming capabilities through wireless accessories. Streamed auditory signals may include music, environmental sounds, noises, and even books. Environmental sounds such as fish tanks, fan noise, waterfalls, television, and radio may be used to effectively manage tinnitus.

Widex is one hearing aid manufacturer with a U.S. Food and Drug Administration (FDA)-cleared device for tinnitus management called the Zen program. Under the tutelage of Robert Sweetow, Zen therapy includes supporting materials for CBI, an approach implementing cognitive behavioral awareness. In CBI, patients are divided into five categories based on inventory scores. These categories determine how the Zen is programmed. ${ }^{16} \mathrm{Sim}$ ilarly, GN ReSound has published a reference guide and supporting materials for use with their combination devices, which includes educational information on tinnitus and tinnitus management. At the time of this writing, Starkey, Phonak, Siemens, Unitron, General Hearing Instruments, and Amplisound now provide tinnitus and/or combination devices. Each company has produced documents to assist the audiologist. The audiologist interested in tinnitus management will benefit from searching out these documents and available research supporting these tinnitus devices.

After creating a referral list, there are additional items that belong in the audiologist's tinnitus toolbox. There should be a variety of tinnitus management tools to demonstrate with a tinnitus patient. The various music type therapies should be represented, including PAXX, Serenade, and Neuromonics, with the Oasis, Sanctuary, and soon to be Haven devices, as well as various sound therapy options.

Neuromonics' premier programmable and customized device, the Oasis, will soon be programmable through NOAH via USB, and will offer a new progressive muscle relaxation track (guided meditation) and three additional tracks. The original device had two Baroque tracks and two New Age tracks. Two of the additional tracks will be the baroque style of music and the third track will be nature sounds or a new age track. The clinician will be able to choose four of the seven (two Baroque and two New Age tracks) for downloading into the device. The Haven, a new FDA-cleared second tier device from Neuromonics also will be programmable through NOAH and will offer the phase 1 tracks (including adjustable white therapy noise), a data logging feature, and a balance control for auditory asymmetries. It also will have the progressive muscle relaxation track built into the device. The Neuromonics Sanctuary is an entry-level, noncustomized device, that plays four tracks of music, all with the therapy noise embedded. The Sanctuary is designed for those whose hearing loss falls in specific and symmetrical categories.

The SoundCure Serenade is a commercially available and FDA-approved tinnitus treatment device with four therapy tracks. Two tracks are S-Tones, which are unique to SoundCure and are based on personalized pitch and loudness matching procedures; the additional tracks offer narrowband and white noise. There is an independent control for each ear for which any auditory asymmetries can be compensated. The device also is customized based on hearing thresholds and loudness discomfort levels. ${ }^{29}$

The PAXX tinnitus device is made by the Tinnitus Laboratory in collaboration with the Montreal Tinnitus Clinic. The customized earlevel device produces intermittent sounds in the region of hearing loss to promote tinnitus habituation. The suggested paradigm of use is increasing length of passive listening from 10 to 30 minutes four to six times daily. 
The audiologist's tinnitus toolbox should include a sample of many of the devices mentioned previously. It is impossible to predict what sound a patient will prefer. Additionally, the tinnitus toolbox should include tools that may be beneficial for misophonia and hyperacusis management. In cases of hyperacusis, a sound generator worn over a prolonged amount of time is helpful in allowing the patient to acclimatize to the offensive sounds.

It is helpful to have some distraction tools or tricks in the toolbox to reduce the concentration and attention being given to the tinnitus. Even simple things such as stress balls have found their way into our toolbox. Amass a variety of programs to assist the tinnitus patient in relaxation. Techniques such as guided imagery, progressive relaxation, and deep breathing exercises can facilitate both stress and anxiety reduction. Lists of $\mathrm{Web}$ sites that might be helpful should be in the toolbox (see Appendix A). For example, the ATA has a sound mixer on its Web site, which allows the patient to select a combination of sounds that can be mixed and exported to an electronic file and replayed later. Search and compile a list of apps for sound therapy and relaxation that are available for smartphones and tablets. Try them out with patients and at home, and finetune this list often. Often these apps are free and patients appreciate this simple, yet effective option.

There is some evidence that notched music might be helpful for one with tonal tinnitus, if played over the course of a year. ${ }^{30}$ Therefore, guiding the patient on creating their own notched music file, or simply providing them information, is recommended. One can find online demonstrations to learn how to create and record notched versions of one's favorite music. For some patients, the do-it-yourself is not possible, so the toolbox should include the things needed to offer the notched music to patients as a customized tool.

There are vitamins that might be helpful, especially in the case of new tinnitus, and information on these products should find their way into the toolbox. For example, Premier Micronutrient Corporation has a vitamin for hearing health, a product in addition to lip- oflavinoids. Note that the authors are not endorsing these products on evidence-based criteria, only stating that they are available and have worked for some. Information on the various herbal remedies also will find its way into your toolbox; whether or not these are helpful, patients will inquire about them. The clinician must be prepared to discuss whether gingko or dong quai root can be beneficial for tinnitus patients. What are the contraindications to these products? What are the side effects? The information must be as accurate and current as possible, but presented in a positive manner.

The toolbox should include hearing protection. It would certainly behoove the tinnitus patient attending a loud event to protect his or her ears from further damage. At the same time, it must be cautioned that the use, or more specifically, the overuse of ear protectors actually encourages hyperacusis and only should be considered when the level of the sound is truly damaging. Too often we hear that audiologists and physicians are recommending custommade plugs for patients with sound sensitivity to be worn more or less continuously. That is not a situation whereby the patient improves; it only serves to continue to add to the auditory deprivation and increases the sound sensitivity. ${ }^{31}$

\section{SUMMARY}

Tinnitus, hyperacusis, and misophonia are auditory disturbances. They are real, but they do not have to be debilitating. The more the patient feels hopeless, helpless, and out of control, the worse the tinnitus, the hyperacusis, and misophonia become. Learning how to help the patient regain a feeling of control is very powerful for the patient. The more tools we have to offer our patients, the better they will be at managing their tinnitus, their hyperacusis, and their misophonia.

\section{REFERENCES}

1. Heller MF, Bergman M. Tinnitus aurium in normally hearing persons. Ann Otol Rhinol Laryngol 1953;62(1):73-83 
2. Henry J. Audiologic assessment. In: Snow J, ed. Tinnitus Theory and Management. Hamilton, Ontario: BC Decker Inc.; 2004;220;

3. Sheldrake J, Jastreboff M. Role of hearing aids in management of tinnitus. In: Snow J, ed. Tinnitus Theory and Management. Hamilton, Ontario: BC Decker Inc.; 2004:310-313

4. Tyler R, Noble W, Preece J, Dunn C, Witt S. Psychological treatments for tinnitus. In: Snow J, ed. Tinnitus Theory and Management. Hamilton, Ontario: BC Decker Inc.; 2004:314-323

5. Henry J, Wilson P. Cognitive behavioral therapy for tinnitus. In: The Psychological Management of Chronic Tinnitus: A Cognitive Behavioral Approach. Needham Heights, MA: Allyn \& Bacon; 2001:63-94

6. Henry J, Zaugg T, Myers P, et al. Progressive Tinnitus Management. San Diego, CA: Plural Publishing; 2010:8-11

7. Herzfeld M, Kuk F. Clinician's experience with using fractal music for tinnitus management. Hear Rev 2011;18(11):50-55

8. Davis PB, Wilde RA, Steed LG, Hanley PJ. Treatment of tinnitus with a customized acoustic neural stimulus: a controlled clinical study. Ear Nose Throat J 2008;87(6):330-339

9. Johnson R. The masking of tinnitus. In: Vernon J, ed. Tinnitus Treatment and Relief. Boston, MA: Allyn \& Bacon; 1998:164-165

10. Jastreboff P, Hazell J. Tinnitus Retraining Therapy; Implementing the Neurophysiological Model. New York, NY: Cambridge Press; 2004. Ch.3. p. 17

11. Tyler RS, Noble W, Coelho CB, Ji H. Tinnitus retraining therapy: mixing point and total masking are equally effective. Ear Hear 2012;33(5):588-594

12. Goodey R. Introduction to management of tinnitus. In: Moller A, Langguth B, DeRidder D, Kleinjung T, eds. Textbook of Tinnitus. New York, NY: Springer; 2011. Ch.68. p. 524

13. Rauschecker JP, Leaver AM, Mühlau M. Tuning out the noise: limbic-auditory interactions in tinnitus. Neuron 2010;66(6):819-826

14. Henry J, Wilson P. Psychological treatments for tinnitus. In: Vernon J, ed. Tinnitus Treatment and Relief. Boston, MA: Allyn \& Bacon; 1998:99-102

15. Beck J. Cognitive Behavior Therapy: Basics and Beyond. New York, NY: Guilford Press; 2011: 1-17

16. Sweetow R. Widex A/S. Widex Zen Therapy: Managing the Effects of Tinnitus. Copenhagen, Denmark, 2012

17. Searchfield G, Magnusson J, Shakes G, Biesinger E, Kong O. Counseling and psycho-education for tinnitus management. In: Moller A, Langguth B, DeRidder D, Kleinjung T, eds. Textbook of Tinnitus. New York, NY: Springer; 2011:535-555

18. Nagler S. Patient perspective. 2003. Available at: www.tinn.com. Accessed December 4, 2013

19. Nagler S. Letter to a tinnitus sufferer. 2013. Available at: http://www.ata.org/nagler-letter-totinnitus-sufferer. ATA 3:1. Accessed January 8, 2014

20. Henry J, Wilson P. Tinnitus: A Self-Management Guide for the Ringing in Your Ears. Boston, MA: Allyn \& Bacon; 2002. Ch.4. 41-58

21. Tyler R. Tinnitus Treatment: Clinical Protocols. New York, NY: Thieme; 2006;181;

22. Gans J. Mindfulness based tinnitus therapy is an approach with ancient roots. Hear J 2010;63:52-56

23. Tyler R and The University of Iowa. Otolaryngology-Head and Neck Surgery: Tinnitus and Hyperacusis. 2006. Available at: http://www.medicine. uiowa.edu/oto/research/tinnitus/. Accessed December 4, 2013

24. Newman CW, Jacobson GP, Spitzer JB. Development of the tinnitus handicap inventory. Arch Otolaryngol Head Neck Surg 1996;122(2): 143-148

25. Wilson PH, Henry J, Bowen M, Haralambous G. Tinnitus reaction questionnaire: psychometric properties of a measure of distress associated with tinnitus. J Speech Hear Res 1991;34(1):197-201

26. Kuk FK, Tyler RS, Russell D, Jordan H. The psychometric properties of a tinnitus handicap questionnaire. Ear Hear 1990;11(6):434-445

27. Meikle MB, Henry JA, Griest SE, et al. The tinnitus functional index: development of a new clinical measure for chronic, intrusive tinnitus. Ear Hear 2012;33(2):153-176

28. Cronlein T, Geisler P, Hajak G. Introduction to management of tinnitus. In: Moller A, Langguth B, DeRidder D, Kleinjung T, eds. Textbook of Tinnitus. New York, NY: Springer; 2011. Ch.68.505-510

29. Reavis K, Chang J, Zeng F. Patterned sound therapy for the treatment of tinnitus. Hear J 2010;63:21-24

30. Okamoto H, Stracke H, Stoll W, Pantev C. Listening to tailor-made notched music reduces tinnitus loudness and tinnitus-related auditory cortex activity. Proc Natl Acad Sci U S A 2010; 107(3):1207-1210

31. Jastreboff P, Jastreboff M. Hyperacusis. 2001. Available at http://www.audiologyonline.com/articles/hyperacusis-1223. Accessed December 4, 2013 
Appendix A: List of Helpful Web Sites for

Audiologist and Patient Reference (All

Accessed January 15, 2014)

www.ata.org

www.helpguide.org/harvard/mindfulness.htm

http://mindfultinnitusrelief.com/

www.tinn.com

www.medicine.uiowa.edu/oto/research/tinnitus

www.amazon.com

www.neuromonics.com

www.soundcure.com

www.tinnituslab.com

www.melmedtronics.com

www.soundpillow.com

www.bedphones.com

www.sleepphones.com

www.premiermicronutrient.com

http://audacity.sourceforge.net 